\title{
Synthesis of Lignin-based Epoxy Resin using lonic Liquid [BMIm]Cl
}

\author{
Z.L. YANG ${ }^{1}$, R.F.ZHANG ${ }^{1}$, M.Q. CHEN ${ }^{1} \&$ W.T. LI ${ }^{1}$ \\ ${ }^{1}$ School of Chemical Engineering, Anhui University of Science and Technology, Huainan, China
}

KEYWORD: Alkali lignin; Ionic liquid; Propoxylation; Epoxy resin

ABSTRACT: Industrial alkali lignin (LG) was used as raw material and ionic liquid 1-butyl-3methylimidazolium chloride $([\mathrm{BMIm}] \mathrm{Cl})$ was used as reaction system. Alkali lignin was dissolved into the $[\mathrm{BMIm}] \mathrm{Cl}$ and modified as propyl ether lignin(HLG). Then the HLG modified lignin was used to synthesizing the lignin-based epoxy resin (LGEP) with epoxy chloropropane. The structure of LG, HLG and LGEP were characterized with FT-IR, the results indicated that the propyl groups were introduced to the LG and the reaction activity was improved. The expoxy value analysis results showed that the optimum synthesis temperature was $80^{\circ} \mathrm{C}$ and the epoxy value was 0.218 . And the using of $[\mathrm{BMIm}] \mathrm{Cl}$ improved the resin mechanical properties.

\section{INTRODUCTION}

After cellulose, lignin is the secondary abundant renewable natural macromolecule organic polymer in the plant world (Dence 1992), and the annual output of lignin has already reached up to 6 trillion tons (Ragauskas et al. 2014). The main source of alkali lignin is the extracts based on the black liquor in papermaking industry. The utility ratio of alkali lignin is less than tenth because the dispersivity and amorphization of complex lignin structure, which is coupling irregularly via C-C bond and C-O bond by three monomers, guaiacyl, syringyl and p-hydroxy phenyl propane (Jia et al. 2010). But the contained functional groups of lignin, such as methoxyl, aryl-ether link, conjugated double bond, and other active groups have been researched for many lignin applied fields as extraction, hydrolysis, pyrolysis, synthetics recently (Martínez et al. 2008). These multi-functional groups make it possible for lignin to be used as materials to synthesize the polymer materials under suitable reacting condition (Ghaffar \& Fan 2014).

Ionic liquids are used as green solvents in biomass research in the last decade (Pu et al. 2007). It has fine thermostability and chemical stability, and can be separated and recycled easily from lignin solute, which made a new react environment for lignin modified (Fort et al. 2007). These advantages make a distinction between ionic liquid and common organic solvent, and become the research hotspot of lignin green applied technology (Lan et al. 2011).

Epoxy resin (EP) is one kind of thermosetting polymers, and can be widely used for plastics, chemical and mechanical industry after curing (Sathitsuksanoh et al. 2014). The plentiful phenolic and alcoholic hydroxyl group in lignin can be used in epoxidation reaction (Laurichesse \& Avérous 2014), therefore this paper tried to use industrial alkali lignin as raw material to synthesize the lignin-based epoxy resin after chemical modified in ionic liquid 1-butyl-3-methylimdazoliuim chloride $([\mathrm{BMIm}] \mathrm{Cl})$.

\section{EXPERIMENT AND METHODS}

\section{Material preparation}

Alkali lignin(LG) was obtained from a papermaking factory in Suzhou City (Anhui Province, China) was milled and sieved to get a powder with particle sizes of $0.2-0.4 \mathrm{~mm}$ after air dried. Its ultimate analysis and proximate analysis were measured according to Chinese Industrial Standard NY/T 1881-2010 (Yang et al. 2014). The elements C, H, O, N, and S in ultimate analysis results are $54.13 \%, 5.13 \%, 36.18 \%, 1.05 \%$, and $3.51 \%$, and the moisture, ash, volatiles, and fixed carbon in proximate analysis are $4.38 \%, 8.69 \%, 49.63 \%$ and $37.30 \%$.

Epoxypropane, epoxy chloropropane, propylene glycol, isopropanol, and hydrochloric acid were purchased from Sinopharm Chemical Reagent Co. Ltd. All the reagents were analytical reagent(AR) 
and used as received. The ionic liquid 1-butyl-3-methylimdazoliuim chloride ([BMIm]Cl, purity $>$ 99\%) was synthesized and characterization in another research paper (Chen et al. 2014).

\section{Lignin purification}

The alkali lignin was dissolved in $\mathrm{NaOH}$ firstly, and washed with distilled water to neutral after precipitated by sulphuric acid. The solid was ground into powder under $0.3 \mathrm{~mm}$. Then the powder was dried at $105^{\circ} \mathrm{C}$ for $8-10$ hours in constant weight as standby.

\section{Lignin dissolution}

The $0.2 \mathrm{~g}$ purified lignin powder was placed into the hydrothermal reaction vessel, then sealed the reactor after added $6 \mathrm{~mL}$ ionic liquid [BMIm] Cl. Stirring the mixture at $75^{\circ} \mathrm{C}$ for 3 hours to modified the alkali lignin, and the mixture was centrifuged to get the liquid after reaction.

\section{Lignin propoxylation}

Set $0.1 \mathrm{~g} \mathrm{KOH}$ as catalyst, $1 \mathrm{~mL}$ propylene glycol as initiating agent and $3 \mathrm{~mL}$ epoxy-propane were added in this modified lignin liquid with $[\mathrm{BMIm}] \mathrm{Cl}$ in order, then lignin propoxylation was occured at $180^{\circ} \mathrm{C}$ for 2 hours in a sealed autoclave. After the reacted mixture was cooled to room temperature (about $25^{\circ} \mathrm{C}$ ), $3 \mathrm{~mL}$ epoxy-propane was added and repeated propoxylation at $180^{\circ} \mathrm{C}$ for 2 hours. At last, the reaction mixture was taken out as propyl ether lignin(HLG) after cooled to room temperature.

Synthesis of lignin-based epoxy resin

$4 \mathrm{~mL}$ epoxy-propane was added in HLG and remainly reacted at a certain temperature for 3 hours. While the reaction was fininshed, the mixture was washed by water after cooled. Then the ligninbased epoxy resin (LGEP) was got for characterization.

\section{Characterization analysis}

Ultraviolet-visible (UV) spectrophotometer (UV-2550) produced from Shimadzu (Japan), Fourier transform infrared (FT-IR) spectrometer (Nicolet 8700) produced from Thermo Scientific Instrument Co. (USA) and scanning electron microscope (SEM, S-3000N) produced from Hitachi (Japan) was used for characterization analysis.

\section{UV absorption spectrum}

The ultraviolet absorption spectrum of alkali lignin which was dissolved in $\mathrm{NaOH}$ was detected by UV spectrophotometer in the wavelength range of 500-200nm.

\section{FT-IR spectra}

FT-IR analysis was used to analyze the structure of LG, HLG and LGEP by KBr plates using liquid membrane method, meanwhile the changes of each functional group was compared. The FT-IR absorption spectra were drawn in the spectral range of $4000-400 \mathrm{~cm}^{-1}$ which scanned at room temperature (35 scans).

\section{SEM}

The samples were recorded using a Hitachi S-3000N scanning electron microscope (SEM) with the accelerating voltage $28 \mathrm{kV}$ at 400 times magnification.

\section{Determination of epoxy value}

The isopropanol hydrochloride method was used to measure epoxy value of LGEP under different temperature to explore the optimum temperature of the reaction. 


\section{Determination method}

The $0.2 \mathrm{~g}$ sample of LGEP was placed into $250 \mathrm{~mL}$ conical beaker, and then $10 \mathrm{~mL}$ isopropanolhydrochloride acid ( $\mathrm{V}: \mathrm{V}=40: 1)$ mixed solvent was added. After dissoluting 10min, 3 drops of methyl red was added into this mixture as acid-base indicator. Via using $0.1 \mathrm{~mol} / \mathrm{L}$ sodium hydroxide standard titration solution under magnetic stirring, the mixture would become yellow at titration end-point. The consuming volume of $\mathrm{NaOH}$ standard titration solution would be recorded for calculation. Repeat the same method to determine the blank sample.

\section{Calculation of epoxy value}

The epoxy value of LGEP can be calculated by following equation (1):

$$
\text { Epoxy value }=\frac{\left(V_{0}-V\right) * C}{10 \mathrm{~m}}
$$

where $V_{0}=$ the volume of $\mathrm{NaOH}$ consumed in blank test which was used by the unit of $\mathrm{mL} ; V=$ the volume of $\mathrm{NaOH}$ consumed in sample test which was also used $\mathrm{mL}$ as unit; $C=$ the concentration of standard solution of sodium hydroxide with the unit of $\mathrm{mol} / \mathrm{L} ; \mathrm{m}=$ the weight of the LGEP sample with the unit of $g$.

\section{RESULTS AND DISCUSSION}

\section{The UV spectrum analysis of alkali lignin}

The ultraviolet absorption spectrum of alkali lignin is shown in Figure 1. It can be seen that there is a strong absorption peak at $282 \mathrm{~nm}$.

In the ultraviolet characteristic absorption peak of lignin, absorption peaks of aromatic ring common appears around $280 \mathrm{~nm}$, and it can be identified as the guaiacyl monomer unit of alkali lignin because the raw material was constituted major of guaiac wood base constitutional unit.

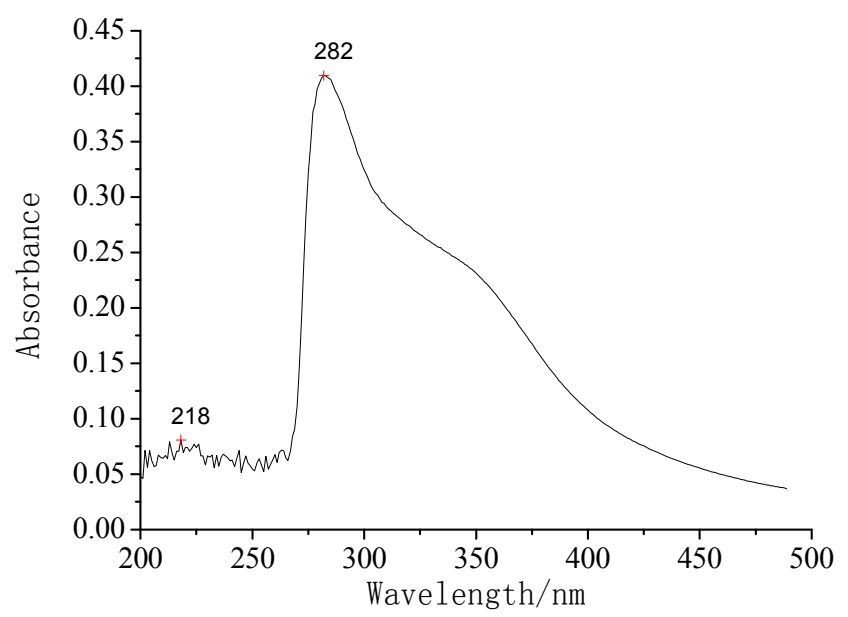

Figure 1. The UV spectrum of alkali lignin.

\section{FT-IR spectra analysis of LG, HLG and LGEP}

The contrast FT-IR spectra between the three of LG, HLG and LGEP were shown in Figure 2. Hydroxyl characteristic peak appeared nearby $3400 \mathrm{~cm}^{-1}$, and the increase intensity of peaks around the $3173 \mathrm{~cm}^{-1}$ and $2987 \mathrm{~cm}^{-1}$ is the stretching vibration characteristic peak of methyl(- $\left.\mathrm{CH}_{3}\right)$. Near 2829 $\mathrm{cm}^{-1}$ the stretching vibration peaks of methylene $\left(-\mathrm{CH}_{2}-\right)$ enhanced obviously and the deformation vibration peaks of methyl $\left(-\mathrm{CH}_{3}\right)$ and methyne $(\equiv \mathrm{CH})$ appeared respectively near $1373 \mathrm{~cm}^{-1}$ and 1330 $\mathrm{cm}^{-1}$ showing that the number of methyl, methylene, methyne significantly increased, which showed that HLG chain length has increased significantly. The skeleton vibration peak of aromatic ring appeared in $1610 \mathrm{~cm}^{-1}, 1510 \mathrm{~cm}^{-1}$ and $1143 \mathrm{~cm}^{-1}$.

Beside in the FT-IR spectrum of HLG, the stretching vibration characteristic peak of ether bond (CO-C) appeared nearby $1180 \mathrm{~cm}^{-1}$ shows that high activity of hydroxyl in lignin has attended the 
reaction. In the FT-IR spectrum of LGEP, the stretching vibration characteristic peak of the epoxy group appeared near $946 \mathrm{~cm}^{-1}$ which occurred a significant hypochromatic shift from $910 \mathrm{~cm}^{-1}$ means that the epoxidation reaction of HLG has been successful import and a stability resin structure has been generated.

Compared with the spectra of LG and HLG, the spectrum of LGEP appeared stretching absorption peak of the epoxy group obviously near $926 \mathrm{~cm}^{-1}$, which meaning that the successful grafting of epoxy group in lignin. The significant decrease of stretching vibration absorption peak and the narrow of the peak width from $3400 \mathrm{~cm}^{-1}$ to $3300 \mathrm{~cm}^{-1}$, and also the obvious weakness of the $\mathrm{O}-\mathrm{H}$ plane bending vibration absorption peak near $1414 \mathrm{~cm}^{-1}$, and the obvious enhancement of the attachment ether bond absorption peak after the reaction all show that high activity hydroxy of HLG involved into the reaction that generated ether bonds.

Furthermore, the obvious enhancement of absorption peak of $1035 \mathrm{~cm}^{-1}$ shows that high activity of hydroxyl in lignin has attended the chain reaction.

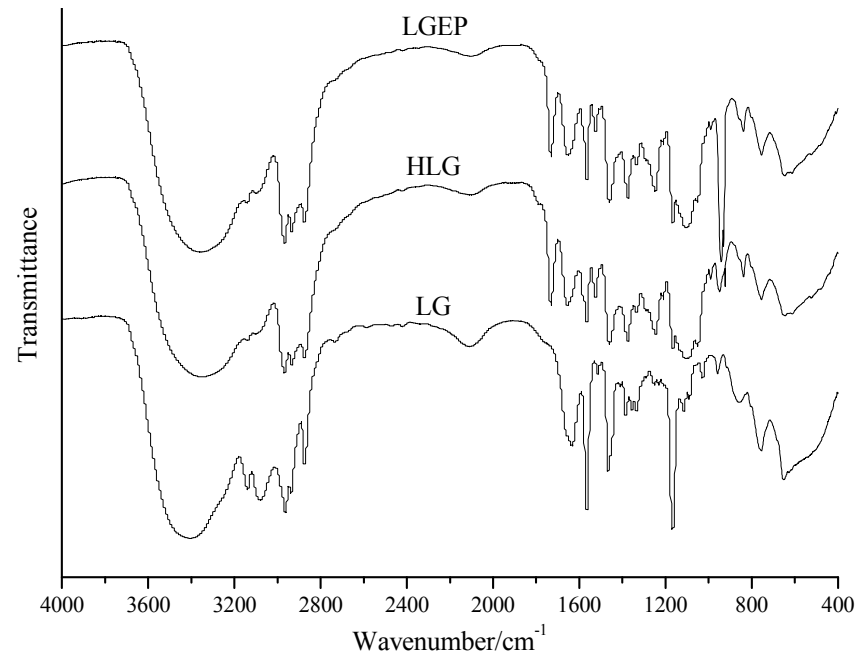

Figure 2. The FT-IR spectra of LG, HLG and LGEP.

\section{The morphologies of LG and HLG}

To show the effect of different substitutions on the surface morphology, SEM images of the LG and HLG samples are shown in Figure 3.
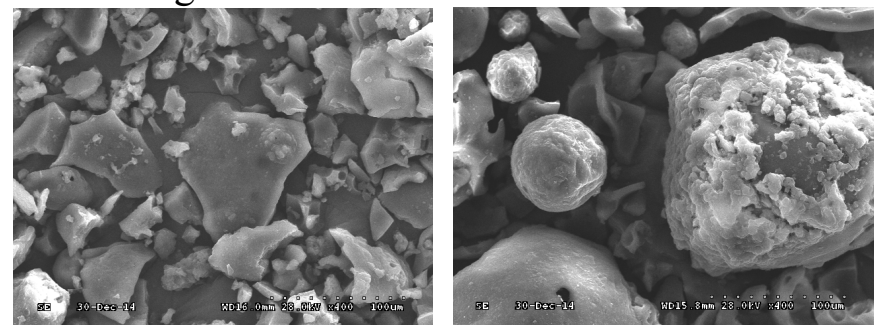

Figure 3. The SEM images of LG(left) and HLG(right).

By using $[\mathrm{BMIm}] \mathrm{Cl}$, the propyl ether lignin has became rounded which make a distinction between the crystalloid raw lignin. HLG has polyporous surface morphology.

\section{Determination of epoxy value}

The isopropanol hydrochloride method was used to measure epoxy value of LGEP generated at different temperature of $60^{\circ} \mathrm{C}, 80^{\circ} \mathrm{C}$ and $100^{\circ} \mathrm{C}$.

By using the isopropanol-hydrochloride acid determinnation method metioned before (see in 2.3), the results of LGEP epoxy value are calculated as follows: the epoxy value of LGEP generated at different temperature of $60^{\circ} \mathrm{C}, 80^{\circ} \mathrm{C}$ and $100^{\circ} \mathrm{C}$ are matched along with $0.206,0.218$, and 0.209 , respectively. And the optimum synthesis temperature was ascertained at $80^{\circ} \mathrm{C}$ by the epoxy value.

\section{The mechanical properties of LGEP}

The lignin-based epoxy resin (LGEP) sample synthetized using [BMIm] $\mathrm{Cl}$ at $80^{\circ} \mathrm{C}$ was photoed in Figure 3. The dynamic viscosity of this sample is 33Pa.S and the tensile strength of this sample is 
$15 \mathrm{MPa}$, higher than the samples without using [BMIm] Cl which only at $25 \mathrm{~Pa} . \mathrm{S}$ and $9 \mathrm{MPa}$. The results shown that mechanical properties of this LGEP sample was improved.

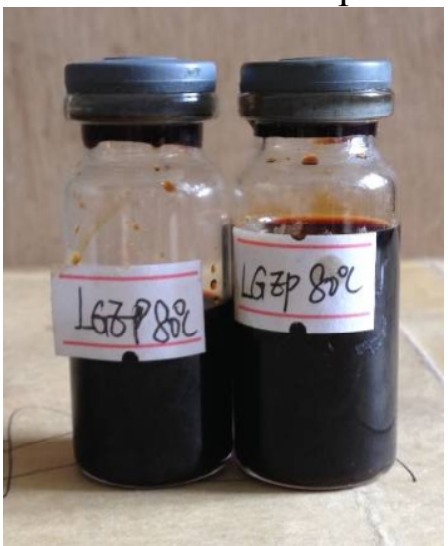

Figure 4. The lignin-based epoxy resin (LGEP) sample.

\section{CONCLUSIONS}

Alkali lignin was used as raw material and ionic liquid [BMIm] $\mathrm{Cl}$ was used as reaction media in this research. Modification of lignin and the synthesis of epoxy resin was proceed in [BMIm]Cl. The result of FT-IR spectra analysis indicated that the propyl group was imported to the LG. In the case of raw materials and catalyst dosage remained constantly and with epoxy value as the reference standard, the effect of temperature on the synthesis of epoxy resin was studied. The conclusion shows that the best reaction temperature was $80^{\circ} \mathrm{C}$ and the epoxy value was 0.218 . And the using of $[\mathrm{BMIm}] \mathrm{Cl}$ improved the resin mechanical properties.

\section{ACKNOWLEDGEMENTS}

The authors gratefully acknowledge the financial support of the National Natural Science Foundation of China (Grant No. 21376007), the National Key Technology R\&D Program of China (Grant No. 2014BAD02B03), the Scientific Research Foundation for Talents Project of Anhui University of Science and Technology and the Training Programs of Innovation and Entrepreneurship for Undergraduates of Anhui Educational Committee of China (Grant No. AH201410361214).

\section{REFERENCES}

[1] Chen, M.Q., Yang, Z.L., Zhang, W.T., \& Cao, W.W. 2014. Synthesis and characterization of 1butyl-3-methyl imidazolium chloride. Journal of Anhui University of Science \& Technology, 34(2), $1-4$.

[2] Dence, C.W. 1992. General structure features of lignins In Lin, E.S., and Dence, C.W. (eds.), Methods in Lignin Chemistry, 3-6. Berlin, Heidelberg: Springer-Verlag.

[3] Fort, D.A., Remsing, R.C., Swatloski, R.P., Moyna, P., Moyna, G., \& Rogers, R.D. 2007. Can ionic liquids dissolve wood? processing and analysis of lignocellulosic materials with 1-n-butyl-3methylimidazolium chloride. Green Chemistry, 9(1), 63-69.

[4] Ghaffar, S.H., \& Fan, M. 2014. Lignin in straw and its applications as an adhesive. International Journal of Adhesion \& Adhesives, 48(1), 92-101.

[5] Jia, S.Y., Cox B.J., Guo X.W., Zhang, Z.C., \& Ekerdt, J.G. 2010. Cleaving the beta-O-4 bonds of lignin model compounds in an acidic ionic liquid, 1-H-3-methylimidazolium chloride: An optional strategy for the degradation of lignin. ChemSusChem, 3(9), 1078-1084.

[6] Lan, W., Liu, C.F., \& Sun, R.C. 2011. Fractionation of bagasse into cellulose, hemicelluloses, and lignin with ionic liquid treatment followed by alkaline extraction.. Journal of Agricultural \& Food Chemistry, 59(16), 8691-8701. 
[7] Laurichesse, S., \& Avérous, L. 2014. Chemical modification of lignins: towards biobased polymers. Progress in Polymer Science, 39(7), 1266-1290.

[8] Martínez A.T., Rencoret J., Marques G., Gutiérrez A., Ibarra D., Jiménez-Barbero J., \& del Río J.C. 2008. Monolignol acylation and lignin structure in some nonwoody plants: a $2 \mathrm{~d} \mathrm{nmr}$ study. Phytochemistry, 69(16), 2831-2843.

[9] Pu, Y., Jiang, N., \& Ragauskas, A.J. 2007. Ionic liquid as a green solvent for lignin. Journal of Wood Chemistry \& Technology, 27(1), 23-33.

[10] Ragauskas, A.J., Beckham, G.T., Biddy, M.J., Chandra, R., Chen, F., \& Davis, M. F., et al. 2014. Lignin valorization: improving lignin processing in the biorefinery. Science, 344(6185): 1246843-1246843.

[11] Sathitsuksanoh, N., Holtman, K.M., Yelle, D.J., Morgan, T., Stavila, V., \& Pelton, J., et al. 2014. Lignin fate and characterization during ionic liquid biomass pretreatment for renewable chemicals and fuels production. Green Chemistry, 16(3), 1236-1247.

[12] Yang, Z.L., Chen, M.Q., Luan, Z.Y., \& Zhang, W.T. (2014). Solubility of alkali lignin in dilute solutions of $[\mathrm{Bmim}] \mathrm{Cl}$ at room temperature. Asian Journal of Chemistry, 26(6), 1707-1710. 